\title{
Association between obesity and polymorphisms in SEC16B, TMEM18, GNPDA2, BDNF, FAIM2 and $M C 4 R$ in a Japanese population
}

Kikuko Hotta $^{1}$, Michihiro Nakamura ${ }^{1}$, Takahiro Nakamura ${ }^{2,3}$, Tomoaki Matsuo ${ }^{4}$, Yoshio Nakata ${ }^{4}$, Seika Kamohara ${ }^{5}$, Nobuyuki Miyatake ${ }^{6}$, Kazuaki Kotani ${ }^{7}$, Ryoya Komatsu ${ }^{8}$, Naoto Itoh $^{9}$, Ikuo Mineo ${ }^{10}$, Jun Wada $^{11}$, Hiroaki Masuzaki ${ }^{12}$, Masato Yoneda ${ }^{13}$, Atsushi Nakajima ${ }^{13}$, Tohru Funahashi ${ }^{7}$, Shigeru Miyazaki ${ }^{14}$, Katsuto Tokunaga ${ }^{15}$, Manabu Kawamoto ${ }^{16}$, Takato Ueno ${ }^{17}$, Kazuyuki Hamaguchi ${ }^{18}$, Kiyoji Tanaka ${ }^{4}$, Kentaro Yamada ${ }^{19}$, Toshiaki Hanafusa ${ }^{20}$, Shinichi Oikawa ${ }^{21}$, Hironobu Yoshimatsu ${ }^{22}$, Kazuwa Nakao ${ }^{12}$, Toshiie Sakata $^{22}$, Yuji Matsuzawa ${ }^{7}$, Naoyuki Kamatani ${ }^{3}$ and Yusuke Nakamura ${ }^{23}$

There is evidence that the obesity phenotype in the Caucasian populations is associated with variations in several genes, including neuronal growth regulator 1 (NEGR1), SEC16 homolog B (SCE16B), transmembrane protein 18 (TMEM18), ets variant 5 (ETV5), glucosamine-6-phosphate deaminase 2 (GNPDA2), prolactin (PRL), brain-derived neurotrophic factor (BDNF), mitochondrial carrier homolog 2 (MTCH2), Fas apoptotic inhibitory molecule 2 (FAIM2), SH2B adaptor protein 1 (SH2B1), v-maf musculoaponeurotic fibrosarcoma oncogene homolog (MAF), Niemann-Pick disease, type C1 (NPC1), melanocortin 4 receptor (MC4R) and potassium channel tetramerisation domain containing 15 (KCTD15). To investigate the relationship between obesity and these genes in the Japanese population, we genotyped 27 single-nucleotide polymorphisms (SNPs) in 14 genes from obese subjects $(n=1129$, body mass index $(\mathrm{BMI}) \geqslant 30 \mathrm{~kg} \mathrm{~m}^{-2}$ ) and normal-weight control subjects $\left(n=1736, \mathrm{BMI}<25 \mathrm{~kg} \mathrm{~m}^{-2}\right)$. The SNP rs 10913469 in SEC16B ( $P=0.000012)$ and four SNPs (rs2867125, rs6548238, rs4854344 and rs7561317) in the TMEM18 gene $(P=0.00015)$, all of which were in almost absolute linkage disequilibrium, were significantly associated with obesity in the Japanese population. SNPs in GNPDA2, BDNF, FAIM2 and MC4R genes were marginally associated with obesity $(P<0.05)$. Our data suggest that some SNPs identified by genome-wide association studies in the Caucasians also confer susceptibility to obesity in Japanese subjects.

Journal of Human Genetics (2009) 54, 727-731; doi:10.1038/jhg.2009.106; published online 23 October 2009

Keywords: BDNF gene; FAIM2 gene; GNPDA2 gene; Japanese; MC4R gene; obesity; SCE16B gene; TMEM18 gene

\section{INTRODUCTION}

Obesity is the most common nutritional disorder in developed countries and is a major risk factor for hypertension, cardiovascular disease and type II diabetes. ${ }^{1,2}$ Genetic factors, as well as environmental factors, contribute to the development of obesity. ${ }^{3-5}$ As a result of recent progress in single-nucleotide polymorphism (SNP) genotyping techniques, it is now possible to conduct genome-wide screens to identify common genetic variants associated with obesity. We carried out a large-scale case-control association study and found that secretogranin III $(S C G 3)^{6}$ and myotubularin-related protein 9 $(M T M R 9)^{7}$ confer susceptibility to the obesity phenotype in the Japanese population. Genome-wide association studies have shown

${ }^{1}$ Laboratory for Endocrinology and Metabolism, Center for Genomic Medicine, RIKEN, Yokohama, Japan; ${ }^{2}$ Laboratory for Mathematics, National Defense Medical College, Tokorozawa, Japan; ${ }^{3}$ Laboratory for Statistical Analysis, Center for Genomic Medicine, RIKEN, Tokyo, Japan; ${ }^{4}$ Graduate School of Comprehensive Human Sciences, University of Tsukuba, Tsukuba, Japan; ${ }^{5}$ Health Science University, Yamanashi, Japan; ${ }^{6}$ Okayama Southern Institute of Health, Okayama, Japan; ${ }^{7}$ Department of Metabolic Medicine, Graduate School of Medicine, Osaka University, Osaka, Japan; ${ }^{8}$ Rinku General Medical Center, Osaka, Japan; ${ }^{9}$ Toyonaka Municipal Hospital, Osaka, Japan; ${ }^{10}$ Otemae Hospital, Osaka, Japan; ${ }^{11}$ Department of Medicine and Clinical Science, Okayama University Graduate School of Medicine and Dentistry, Okayama, Japan; ${ }^{12}$ Department of Medicine and Clinical Science, Kyoto University Graduate School of Medicine, Kyoto, Japan; ${ }^{13}$ Division of Gastroenterology, Yokohama City University Graduate School of Medicine, Yokohama, Japan; ${ }^{14}$ Tokyo Postal Services Agency Hospital, Tokyo, Japan; ${ }^{15}$ Itami City Hospital, Hyogo, Japan; ${ }^{16}$ Institute of Rheumatology, Tokyo Women's Medical University, Tokyo, Japan; ${ }^{17}$ Research Center for Innovative Cancer Therapy, Kurume University, Kurume, Japan; ${ }^{18}$ Department of Community Health and Gerontological Nursing, Faculty of Medicine, Oita University, Oita, Japan; ${ }^{19}$ Division of Endocrinology and Metabolism, Department of Medicine, Kurume University, Kurume, Japan; ${ }^{20}$ First Department of Internal Medicine, Osaka Medical College, Osaka, Japan; ${ }^{21}$ Division of Endocrinology and Metabolism, Department of Medicine, Nippon Medical School, Tokyo, Japan; ${ }^{22}$ Department of Internal Medicine I, Faculty of Medicine, Oita University, Oita, Japan and ${ }^{23}$ Laboratory for Molecular Medicine, Human Genome Center, The Institute of Medical Science, University of Tokyo, Tokyo, Japan

Correspondence: Dr K Hotta, Laboratory for Endocrinology and Metabolism, Center for Genomic Medicine, RIKEN, 1-7-22 Suehiro, Tsurumi-ku, Yokohama, Kanagawa 230-0045, Japan. 
that variations in the upstream region of the insulin induced gene 2 $(\text { INSIG2 })^{8}$ and in the fat mass and obesity associated $(F T O)^{9-11}$ genes are linked with the obesity phenotype. We recently reported the association between variations in the FTO and INSIG2 genes and obesity in Japanese subjects. ${ }^{12,13}$ Large-scale genome-wide association studies have recently shown that 16 loci are related to variations in body mass index (BMI). ${ }^{14-17}$ With the exception of FTO, these loci have not been examined in Asian populations.

To investigate the relationship between obesity and SNPs in the 15 loci in the Japanese population, we carried out a case-control association study using patients with adult obesity (BMI $\geqslant 30 \mathrm{~kg} \mathrm{~m}^{-2}$ ) and normal-weight controls (BMI $<25 \mathrm{~kg} \mathrm{~m}^{-2}$ ). This study found that rs10913469 in the SEC16 homolog B (SCE16B) gene and four SNPs (rs2867125, rs6548238, rs4854344 and rs7561317) in the transmembrane protein 18 (TMEM18) gene were associated with adult obesity.

\section{MATERIALS AND METHODS}

\section{Study subjects}

The sample size of the obese Japanese subjects (BMI $\geqslant 30 \mathrm{~kg} \mathrm{~m}^{-2}$ ) was 1129 (male/female ratio, 571:558; age, $49.1 \pm 13.6$ years; BMI, $34.2 \pm 5.3 \mathrm{~kg} \mathrm{~m}^{-2}$ ), whereas for the Japanese normal-weight controls (BMI $<25 \mathrm{~kg} \mathrm{~m}^{-2}$ ), it was 1736 (male/female ratio, 849:887; age, $48.8 \pm 15.8$ years; BMI, $21.7 \pm 2.1 \mathrm{~kg} \mathrm{~m}^{-2}$ ). The obese subjects were recruited from among outpatients of 15 medical institutes. Patients with secondary obesity, obesity-related hereditary disorders or medication-induced obesity were not included in the study. The normalweight control subjects were recruited from among subjects who had undergone a medical examination for the screening of common diseases at eight medical institutes. Self-claiming nationality of all subjects were Japanese. As most Japanese individuals fell into two clusters, Hondo and Rykyu, ${ }^{18}$ we collected the case and control subjects at the region of Hondo (Kyushu, Chugoku-Shikoku, Kinki and Kanto-Koshinetsu). Details of the geographical region of the subjects was described in Supplementary Table 1. The clinical features of the subjects are summarized in Table 1. Each subject gave written informed consent, and the protocol was approved by the ethics committee of each institution and that of RIKEN.

\section{DNA preparation and SNP genotyping}

Genomic DNA was prepared using Genomix (Talent Srl, Trieste, Italy) from blood samples collected from each subject. We constructed Invader probes (Third Wave Technologies, Madison, WI, USA) for rs3101366 and rs2815752 in the neuronal growth regulator 1 (NEGR1) gene; rs6548238 and rs4854344 in the TMEM18 gene; rs10938397 in the glucosamine-6-phosphate deaminase 2 (GNPDA2) gene; rs4074134, rs4923461, rs925946 and rs6265 in the brainderived neurotrophic factor $(B D N F)$ gene; rs10838738 in the mitochondrial carrier homolog 2 (MTCH2) gene; rs8049439, rs4788102 and rs7498665 in the SH2B adaptor protein 1 (SH2B1) gene; rs1424233 in the v-maf musculoaponeurotic fibrosarcoma oncogene homolog (MAF) gene; rs1805081 in the Niemann-Pick disease, type C1 (NPC1) gene; rs17782313 in the melanocortin 4 receptor (MC4R) gene; and rs29941 and rs1 1084753 in the potassium channel tetramerisation domain containing 15 (KCTD15) gene. The SNPs were genotyped using Invader assays as previously described. ${ }^{19,20}$ Pre-designed TaqMan probes (Applied Biosystems, Foster City, CA, USA) were purchased for genotyping of rs2568958 (C_1682788_10) in the NEGR1 gene, rs4712652 (C_29580306_10) in the prolactin (PRL) gene, rs10508503 (C_29615408_20) in the phosphotriesterase related (PTER) gene, rs10501087 (C_1751776_10) in the BDNF gene, rs7138803 (C_29248155_10) in the Fas apoptotic inhibitory molecule 2 (FAIM2) gene and rs12970134 (C_3058722_10) in the MC4R gene. TaqMan probes for rs10913469 in the SEC16B gene, rs2867125 and rs7561317 in the TMEM18 gene, and rs7647305 in the ets variant 5 (ETV5) gene were designed and synthesized by Applied Biosystems. The success rates of these assays were $>99.0 \%$, with the exception of rs 10501087 (98.9\%) and rs12970134 (96.3\%) in the control subjects (Supplementary Table 2). Using rs11084753 and rs7498665, we confirmed that there was almost a $100 \%$ agreement between the results of genotyping by Invader and Taqman assays.

\section{Statistical analysis}

For each case-control study, the $\chi^{2}$-test (additive model) was performed according to Sladek et al. ${ }^{21}$ We coded genotypes as 0,1 and 2, depending on the number of copies of the risk alleles. Odds ratios (ORs) adjusted for age and gender were calculated using multiple logistic regression analysis with genotypes, age and gender as the independent variables.

The Hardy-Weinberg equilibrium was assessed using the $\chi^{2}$-test. ${ }^{22}$ We used the linkage disequilibrium (LD) measures $D^{\prime}$ and $r^{2}$, calculated as previously reported. $^{23}$ Although we collected the samples at the region of Hondo, we performed Wright's F-statistics ${ }^{24}$ to evaluate the difference in the population structures of our case and control groups using randomly selected 28 SNPs. The results indicated that the population structure of our case and control groups were almost the same in view of a very small $F_{\mathrm{ST}}$ value between the both groups $\left(F_{\mathrm{ST}}=0.00011\right)$. Simple comparison of the clinical data between case and control groups was carried out using the Mann-Whitney $U$-test. Simple comparison of the clinical data in the different genotypes was carried out using the Kruskal-Wallis test.

\section{RESULTS}

The most significant association was observed for rs10913469 in the $S E C 16 B$ gene $(P=0.000012$, allele-specific OR ( $95 \%$ confidence interval (CI)) adjusted for age and gender=1.31 (1.16-1.48)). Four SNPs (rs2867125, rs6548238, rs4854344 and rs7561317) in the TMEM18 gene were also associated significantly with obesity in the Japanese subjects (Table 2). Four SNPs in the TMEM18 gene were in

Table 1 Clinical characteristics of the subjects

\begin{tabular}{|c|c|c|c|c|c|}
\hline & \multicolumn{2}{|c|}{ Case } & \multicolumn{2}{|c|}{ Control } & \multirow[b]{2}{*}{ P-value } \\
\hline & $n$ & Value & $\mathrm{n}$ & Value & \\
\hline Gender (male/female) & 1129 & $571 / 558$ & 1736 & $849 / 887$ & 0.38 \\
\hline Age (years) & 1129 & $49.1 \pm 13.6$ & 1736 & $48.8 \pm 15.8$ & 0.37 \\
\hline $\mathrm{BMI}\left(\mathrm{kg} \mathrm{m}^{-2}\right)$ & 1129 & $34.2 \pm 5.3$ & 1736 & $21.7 \pm 2.1$ & $<0.0001$ \\
\hline Fasting glucose (mg per $100 \mathrm{ml}$ ) & 1085 & $124.8 \pm 47.0$ & 1731 & $98.7 \pm 22.9$ & $<0.0001$ \\
\hline Total cholesterol (mg per $100 \mathrm{ml}$ ) & 1101 & $210.8 \pm 38.4$ & 1731 & $203.3 \pm 36.6$ & $<0.0001$ \\
\hline Triglycerides (mg per $100 \mathrm{ml}$ ) & 1093 & $157.4 \pm 109.4$ & 1731 & $106.8 \pm 74.1$ & $<0.0001$ \\
\hline HDL cholesterol (mg per $100 \mathrm{ml}$ ) & 1007 & $53.0 \pm 18.0$ & 1691 & $64.2 \pm 15.5$ & $<0.0001$ \\
\hline Systolic blood pressure (mm Hg) & 1056 & $136.7 \pm 18.4$ & 1693 & $123.6 \pm 17.9$ & $<0.0001$ \\
\hline Diastolic blood pressure $(\mathrm{mm} \mathrm{Hg})$ & 1056 & $84.5 \pm 12.5$ & 1693 & $76.2 \pm 11.2$ & $<0.0001$ \\
\hline
\end{tabular}

Abbreviations: BMI, body mass index; HDL, high-density lipoprotein.

Data are shown as the mean \pm s.d. Male/female ratio was analyzed by the $\chi^{2}$-test. $P$-values for quantitative traits were analyzed using the Mann-Whitney $U$-test. 
Table 2 Association of candidate SNP loci with obesity

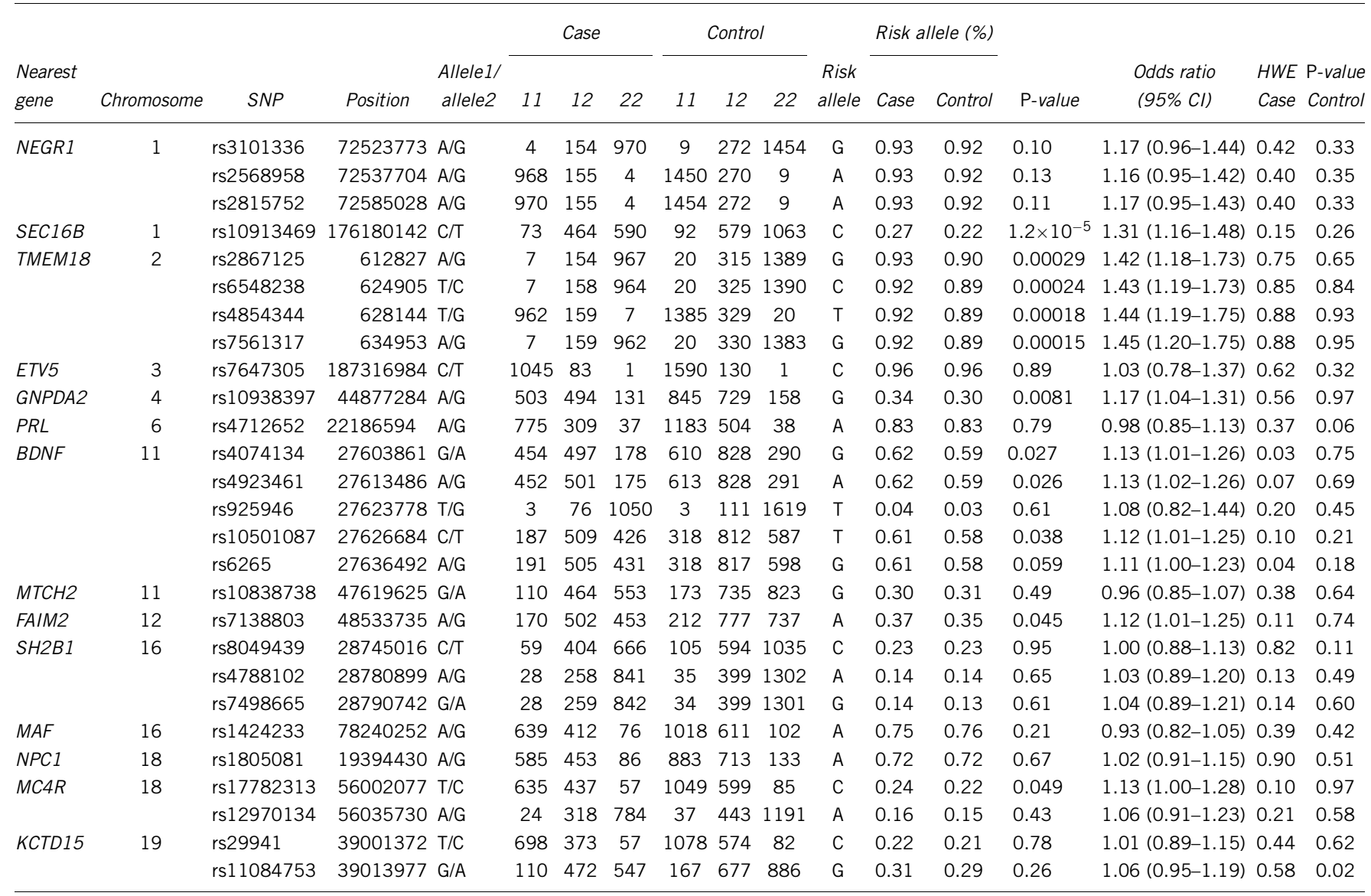

Abbreviations: $\mathrm{Cl}$, confidence interval; SNP, single-nucleotide polymorphism, HWE, Hardy-Weinberg equilibrium.

The odds ratio for each SNP was adjusted simultaneously for age and gender using the additive model.

almost absolute LD $\left(D^{\prime}>0.99, r^{2}>0.95\right)$. The $P$-value of rs7561317 was smallest among the four SNPs $(P=0.00015, \mathrm{OR}=1.45 \quad(95 \%$ $\mathrm{CI}=1.20-1.75))$. SNP rs10938397 in the GNPDA2 gene, three SNPs (rs4074134, rs4923461 and rs10501087) in the BDNF gene, rs7138803 in the FAIM2 gene and rs17782313 in the MC4R gene were marginally associated with obesity $(P<0.05)$. Three SNPs (rs4074134, rs4923461 and rs10501087) in the BDNF gene were in almost complete LD $\left(D^{\prime}>0.99, r^{2}>0.86\right)$. The risk alleles of the above SNPs were the same as reported previously. ${ }^{14-17}$ The other SNPs investigated were not associated with obesity in the Japanese subjects. One SNP, rs10508503, in the PTER gene was monomorphic in the Japanese population. All SNPs were in the Hardy-Weinberg equilibrium $(P>0.05)$, with the exception of rs4074134 $(P=0.03)$ and $\operatorname{rs6265}(P=0.04)$ in the obese subjects and rs11084753 in the control subjects $(P=0.02)$.

As some of these SNPs are known to be associated with type II diabetes, ${ }^{15}$ we investigated whether the genotypes of SNP rs10913469 and rs7561317 were associated with the phenotypes of metabolic disorders. We compared the following parameters among the different genotypes in the cases and controls: BMI; blood pressure; and levels of fasting plasma glucose, total cholesterol, triglycerides and high-density lipoprotein (HDL) cholesterol. The quantitative phenotypes for BMI, fasting plasma glucose, total cholesterol, triglycerides, HDL cholesterol and blood pressure were not found to be significantly associated with the genotypes at rs10913469 and rs7561317 in either the case or control groups (Tables 3 and 4). Thorleifsson et al. ${ }^{15}$ reported that rs7138803 was associated with both obesity and type II diabetes, and therefore we also compared the quantitative traits among the different genotypes in the cases and controls. We could, however, find no association between genotypes and the quantitative traits (data not shown).

\section{DISCUSSION}

In this study, we found that the SNPs in the SEC16B and TMEM18 genes were associated significantly with obesity in the Japanese population. We have previously reported that SNPs in the FTO and INSIG2 genes are associated with obesity in Japanese subjects. ${ }^{12,13}$ Therefore, some of the SNPs identified by genome-wide association studies in the Caucasians also confer susceptibility to obesity in Japanese subjects. Further, the SNP in the PTER gene was only a $\mathrm{C}$ allele in our sample, whereas according to the HapMap data, SNPs in the SCG3 gene are risk alleles only in the Caucasians. Therefore, genetic susceptibility to obesity is likely to differ among ethnic groups.

We could not replicate the association 16 SNPs and obesity in this study. This may be due to the low power of the study, as the ORs were around 1.1 in the Caucasians. ${ }^{15}$ In the simulation study, the power of this test was 0.31 in the following condition; the sizes of the case and the control groups are 1129 and 1736, respectively, OR is 1.1, risk allele frequency is 0.2 and the mode of inheritance is additive (Supplementary Figure 1). Therefore, further studies would be necessary to 
Table 3 Comparison of various quantitative phenotypes among the different genotypes at rs10913469 in obese and control subjects

\begin{tabular}{|c|c|c|c|c|c|c|c|c|}
\hline & \multicolumn{3}{|c|}{ Obese $e^{a}$} & \multicolumn{5}{|c|}{ Controla } \\
\hline & $C C$ & CT & $T T$ & $\mathrm{P}$-value & $C C$ & CT & $T T$ & P-value $\mathrm{b}^{\mathrm{b}}$ \\
\hline Age (year) & $47.7 \pm 15.7$ & $48.4 \pm 13.6$ & $49.7 \pm 13.4$ & 0.11 & $48.9 \pm 15.6$ & $48.7 \pm 15.5$ & $48.8 \pm 15.9$ & 0.99 \\
\hline Glucose (mg per 100 ml) & $129.3 \pm 47.7$ & $122.8 \pm 45.0$ & $125.7 \pm 48.5$ & 0.52 & $105.6 \pm 47.8$ & $98.0 \pm 21.9$ & $98.5 \pm 20.1$ & 0.25 \\
\hline Total cholesterol (mg per $100 \mathrm{ml}$ ) & $210.8 \pm 40.7$ & $211.0 \pm 38.3$ & $210.6 \pm 38.3$ & 0.90 & $203.7 \pm 34.7$ & $203.2 \pm 36.1$ & $203.3 \pm 37.0$ & 0.93 \\
\hline Triglycerides (mg per $100 \mathrm{ml}$ ) & $151.0 \pm 81.7$ & $160.1 \pm 111.9$ & $156.3 \pm 110.6$ & 0.77 & $107.3 \pm 65.0$ & $101.5 \pm 63.1$ & $109.6 \pm 80.2$ & 0.17 \\
\hline $\mathrm{DBP}(\mathrm{mm} \mathrm{Hg})$ & $83.1 \pm 9.8$ & $84.1 \pm 12.5$ & $85.1 \pm 12.7$ & 0.23 & $77.1 \pm 12.5$ & $75.5 \pm 11.3$ & $76.5 \pm 11.1$ & 0.12 \\
\hline
\end{tabular}

Abbreviations: BMI, body mass index; DBP, diastolic blood pressure; HDL, high-density lipoprotein; SBP, systolic blood pressure.

Data are presented as the mean \pm s.d.

The data of each quantitative phenotype from obese and control subjects were compared in the different genotypes at rs10913469 in obese and control subjects.

${ }^{\mathrm{b}} P$-values analyzed using the Kruskal-Wallis test in each group of obese and control subjects.

Table 4 Comparison of various quantitative phenotypes among different genotypes at rs7561317 in obese and control subjects

\begin{tabular}{|c|c|c|c|c|c|c|c|c|}
\hline & \multicolumn{3}{|c|}{ Obese ${ }^{a}$} & \multicolumn{5}{|c|}{ Controla } \\
\hline & $T T$ & $C T$ & $C C$ & $\mathrm{P}$-value $\mathrm{b}^{\mathrm{b}}$ & $T T$ & $C T$ & $C C$ & P-value \\
\hline Age (year) & $49.7 \pm 13.0$ & $48.0 \pm 13.6$ & $49.3 \pm 13.6$ & 0.63 & $47.7 \pm 16.1$ & $49.4 \pm 16.1$ & $48.6 \pm 15.7$ & 0.64 \\
\hline $\mathrm{BMI}\left(\mathrm{kg} \mathrm{m}^{-2}\right)$ & $34.1 \pm 5.1$ & $34.9 \pm 6.3$ & $34.1 \pm 5.1$ & 0.14 & $21.5 \pm 2.1$ & $21.7 \pm 2.1$ & $21.7 \pm 2.0$ & 0.88 \\
\hline Glucose (mg per $100 \mathrm{ml}$ ) & $128.3 \pm 45.0$ & $129.9 \pm 49.3$ & $124.0 \pm 46.7$ & 0.17 & $100.3 \pm 11.6$ & $98.6 \pm 18.3$ & $98.7 \pm 24.1$ & 0.34 \\
\hline Total cholesterol (mg per $100 \mathrm{ml}$ ) & $201.9 \pm 26.1$ & $210.0 \pm 34.2$ & $211.1 \pm 39.1$ & 0.79 & $198.5 \pm 16.5$ & $198.7 \pm 36.3$ & $204.4 \pm 36.8$ & 0.04 \\
\hline Triglycerides (mg per 100 ml) & $108.3 \pm 42.0$ & $155.9 \pm 104.2$ & $158.1 \pm 110.6$ & 0.42 & $121.1 \pm 121.4$ & $103.1 \pm 66.4$ & $107.5 \pm 75.1$ & 0.58 \\
\hline HDL cholesterol (mg per $100 \mathrm{ml}$ ) & $59.7 \pm 22.6$ & $53.9 \pm 30.2$ & $52.8 \pm 14.9$ & 0.44 & $63.6 \pm 10.8$ & $63.0 \pm 15.6$ & $64.4 \pm 15.5$ & 0.51 \\
\hline $\mathrm{SBP}(\mathrm{mm} \mathrm{Hg})$ & $136.3 \pm 5.8$ & $135.2 \pm 16.7$ & $137.0 \pm 18.7$ & 0.66 & $125.6 \pm 15.8$ & $124.0 \pm 17.3$ & $123.5 \pm 18.0$ & 0.61 \\
\hline $\mathrm{DBP}(\mathrm{mm} \mathrm{Hg})$ & $84.7 \pm 7.8$ & $84.4 \pm 11.1$ & $84.5 \pm 12.7$ & 0.93 & $78.1 \pm 11.8$ & $76.6 \pm 10.2$ & $76.1 \pm 11.4$ & 0.38 \\
\hline
\end{tabular}

Abbreviations: BMI, body mass index; DBP, diastolic blood pressure; HDL, high-density lipoprotein; SBP, systolic blood pressure.

Data are shown as the mean \pm s.d.

${ }^{a}$ The data of each quantitative phenotype from obese and control subjects were compared in the different genotypes at rs7561317 in obese and control subjects.

${ }^{b} P$-values analyzed using the Kruskal-Wallis test in each group of obese and control subjects.

elucidate the association between 16 SNPs and obesity in the Japanese population.

The SEC16B encodes long (Sec16L) and short (Sec16S) proteins, which are expressed ubiquitously in various tissues. ${ }^{25}$ Sec16L and Sec16S are required for secretory cargo traffic from the endoplasmic reticulum to the Golgi apparatus. ${ }^{25,26}$ We have reported that SCG3 and secretogranin II (SCG2) form secretory granules with orexin, neuropeptide Y (NPY) and proopiomelanocortin (POMC). ${ }^{6,27}$ Thus, Sec16 may have a role in the transport of SCG3 and SCG2 together with orexin, NPY and POMC from the endoplasmic reticulum to the Golgi apparatus, and also in regulating the secretion of these appetiterelated peptides. In addition to many neuropeptides in the central nervous system, peptides secreted from other tissues, such as adipose tissue and the gastrointestinal organs, participate in the control of food intake. There have been no reports indicating the involvement of Sec16 in appetite regulation, but it is interesting to speculate that Sec16 may be a potential factor in the regulation of food intake and that alteration in the amounts or function of Sec16 could be related to the development of obesity. TMEM18 is also expressed in various tissues, with its expression level being high in the brain and hypothalamus. ${ }^{16}$ This gene may also participate in the appetite-signaling system, although the function of TMEM18 is yet to be elucidated.

Single-nucleotide polymorphisms in the GNPDA2, BDNF, FAIM2 and $M C 4 R$ genes may be associated with obesity in Japanese subjects. As we previously reported, the obesity-related SCG3 and MTMR9 genes are highly expressed in the hypothalamus; ${ }^{6,7}$ the expression of
GNPDA2, BDNF, FAIM2 and MC4R, as well as FTO, is high in the hypothalamus. ${ }^{9,16,28}$ Disruption of BDNF or MC4R gene function in mice results in hyperphagia and obesity, indicating that these genes are involved in the regulation of appetite. ${ }^{29,30}$ Although FAIM2 has an anti-apoptotic function in Hela cells, its role in the hypothalamus is yet to be studied. The function of GNPDA2 in the hypothalamus has also not been elucidated. Although the functions of these genes in the hypothalamus remains to be fully investigated, many of the genes causing monogenic and common obesity are expressed in the hypothalamus, indicating that they have important roles in the regulation of food intake. These genes are therefore likely to confer susceptibility to obesity in Japanese subjects.

In summary, we were able to replicate the previously reported genetic variations in the SEC16B and TMEM18 genes that may influence the risk of obesity in the Japanese population. Genetic variations in GNPDA2, BDNF, FAIM2 and MCAR may be associated with obesity in the Japanese.

\section{CONFLICT OF INTEREST}

The authors declare no conflict of interest.

\section{ACKNOWLEDGEMENTS}

The authors express their appreciation to Ms Ritsuko Kusano for her contribution to our study. This work was supported by a grant from the Japanese Millennium Project and Grants-in-Aid from the Ministry of Education, Science, Sports and Culture of Japan (21591186). 
1 Kopelman, P. G. Obesity as a medical problem. Nature 404, 635-643 (2000).

2 Wilson, P. W. \& Grundy, S. M. The metabolic syndrome: practical guide to origins and treatment: Part I. Circulation 108, 1422-1424 (2003).

3 Maes, H. H., Neale, M. C. \& Eaves, L. J. Genetic and environmental factors in relative body weight and human adiposity. Behav. Genet. 27, 325-351 (1997).

4 Barsh, G. S., Farooqi, I. S. \& O'Rahilly, S. Genetics of body-weight regulation. Nature 404, 644-651 (2000).

5 Rankinen, T., Zuberi, A., Chagnon, Y. C., Weisnagel, S. J., Argyropoulos, G., Walts, B. et al. The human obesity gene map: the 2005 update. Obesity 14, 529-644 (2006).

6 Tanabe, A., Yanagiya, T., lida, A., Saito, S., Sekine, A., Takahashi, A. et al. Functional single-nucleotide polymorphisms in the secretogranin III (SCG3) gene that form secretory granules with appetite-related neuropeptides are associated with obesity. J. Clin. Endocrinol. Metab. 92, 1145-1154 (2007).

7 Yanagiya, T., Tanabe, A., Iida, A., Saito, S., Sekine, A., Takahashi, A. et al. Association of single-nucleotide polymorphisms in MTMR9 gene with obesity. Hum. Mol. Genet 16, 3017-3026 (2007).

8 Herbert, A., Gerry, N. P., McQueen, M. B., Heid, I. M., Pfeufer, A., Illig, T. et al. A common genetic variant is associated with adult and childhood obesity. Science 312, 279-283 (2006).

9 Frayling, T. M., Timpson, N. J., Weedon, M. N., Zeggini, E., Freathy, R. M., Lindgren, C. M. et al. A common variant in the FTO gene is associated with body mass index and predisposes to childhood and adult obesity. Science 316, 889-894 (2007).

10 Scuteri, A., Sanna, S., Chen, W. M., Uda, M., Albai, G., Strait, J. et al. Genome-wide association scan shows genetic variants in the FTO gene are associated with obesityrelated traits. PLoS Genet. 3, 1200-1210 (2007).

11 Hinney, A., Nguyen, T. T., Scherag, A., Friedel, S., Brönner, G., Müller, T. D. et al. Genome wide association (GWA) study for early onset extreme obesity supports the role of fat mass and obesity associated gene (FTO) variants. PLOS ONE 2, e1361-e1365 (2007).

12 Hotta, K., Nakata, Y., Matsuo, T., Kamohara, S., Kotani, K., Komatsu, R. et al. Variations in the FTO gene are associated with severe obesity in the Japanese. J. Hum. Genet. 53, 546-553 (2008).

13 Hotta, K., Nakamura, M., Nakata, Y., Matsuo, T., Kamohara, S., Kotani, K. et al. INSIG2 gene rs7566605 polymorphism is associated with severe obesity in Japanese. J. Hum. Genet. 53, 857-862 (2008).

14 Loos, R. J., Lindgren, C. M., Li, S., Wheeler, E., Zhao, J. H., Prokopenko, I. et al. Common variants near MC4R are associated with fat mass, weight and risk of obesity. Nat. Genet. 40, 768-775 (2008).

15 Thorleifsson, G., Walters, G. B., Gudbjartsson, D. F., Steinthorsdottir, V., Sulem, P., Helgadottir, A. et al. Genome-wide association yields new sequence variants at seven loci that associate with measures of obesity. Nat. Genet. 41, 18-24 (2009).
16 Willer, C. J., Speliotes, E. K., Loos, R. J., Li, S., Lindgren, C. M., Heid, I. M. et al. Six new loci associated with body mass index highlight a neuronal influence on body weight regulation. Nat. Genet. 41, 25-34 (2009).

17 Meyre, D., Delplanque, J., Chèvre, J. C., Lecoeur, C., Lobbens, S., Gallina, S. et al. Genome-wide association study for early-onset and morbid adult obesity identifies three new risk loci in European populations. Nat. Genet. 41, 157-159 (2009).

18 Yamaguchi-Kabata, Y., Nakazono, K., Takahashi, A., Saito, S., Hosono, N., Kubo, M. et al. Japanese population structure, based on SNP genotypes from 7003 individuals compared to other ethnic groups: effects on population-based association studies. Am. J. Hum. Genet. 83, 445-456 (2008).

19 Ohnishi, Y., Tanaka, T., Ozaki, K., Yamada, R., Suzuki, H. \& Nakamura, Y. A highthroughput SNP typing system for genome-wide association studies. J. Hum. Genet. 46, 471-477 (2001)

20 Takei, T., lida, A., Nitta, K., Tanaka, T., Ohnishi, Y., Yamada, R. et al. Association between single-nucleotide polymorphisms in selectin genes and immunoglobulin A nephropathy. Am. J. Hum. Genet. 70, 781-786 (2002).

21 Sladek, R., Rocheleau, G., Rung, J., Dina, C., Shen, L., Serre, D. et al. A gemone-wide association study identifies novel risk loci for type 2 diabetes. Nature 445, 881-885 (2007).

22 Nielsen, D. M., Ehm, M. G. \& Weir, B. S. Detecting marker-disease association by testing for Hardy-Weinberg disequilibrium at a marker locus. Am. J. Hum. Genet. 63 , 1531-1540 (1998).

23 Devlin, B. \& Risch, N. A comparison of linkage disequilibrium measures for fine-scale mapping. Genomics 29, 311-322 (1995).

24 Wright, S. The genetical structure of populations. Ann. Eugen. 15, 323-354 (1951).

25 Bhattacharyya, D. \& Glick, B. S. Two mammalian Sec 16 homologues have nonredundant functions in endoplasmic reticulum (ER) export and transitional ER organization. Mol. Biol. Cell 18, 839-849 (2007).

26 Watson, P., Townley, A. K., Koka, P., Palmer, K. J. \& Stephens, D. J. Sec16 defines endoplasmic reticulum exit sites and is required for secretory cargo export in mammalian cells. Traffic 7, 1678-1687 (2006).

27 Hotta, K., Hosaka, M., Tanabe, A. \& Takeuchi, T. Secretogranin II binds to secretogranin III and forms secretory granules with orexin, neuropeptide Y, and POMC. J. Endocrinol. 202, 111-121 (2009).

28 Somia, N. V., Schmitt, M. J., Vetter, D. E., Van Antwerp, D., Heinemann, S. F. \& Verma, I. M. LFG: an anti-apoptotic gene that provides protection from Fas-mediated cell death. Proc. Natl Acad. Sci. USA 96, 12667-12672 (1999).

29 Unger, T. J., Calderon, G. A., Bradley, L. C., Sena-Esteves, M. \& Rios, M. Selective deletion of Bdnf in the ventromedial and dorsomedial hypothalamus of adult mice results in hyperphagic behavior and obesity. J. Neurosci. 27, 14265-14274 (2007).

30 Huszar, D., Lynch, C. A., Fairchild-Huntress, V., Dunmore, J. H., Fang, Q., Berkemeier, L. R. et al. Targeted disruption of the melanocortin-4 receptor results in obesity in mice. Cel/ 88, 131-141 (1997).

Supplementary Information accompanies the paper on Journal of Human Genetics website (http://www.nature.com/jhg) 